\title{
DTMF Based Irrigation Water Pump Control System
}

\author{
Mandeep Guleria1), Akanksha Rathi2), Deepraj Singh Sajwan3), \\ Jasbir Singh Negi4), Nidhi Chauhan ${ }^{5}$
}

\begin{abstract}
As a result of advancement in technology it became easy for human beings to ease their work and at the same time complete the workwith less human force, less time and with better results. In this project we look forward to telephonic signaling approach using Dual tone multi-frequency technique in controlling the various electrical loads such as irrigation water pumps situated in inaccessible areas. The present work is based on the principle of Dual tone multi-frequency (DTMF) signal received from any mobile phone to switch on-off the desired electrical loads such as in our farms, in factories, tube wells, irrigation water pumps etc. In our agricultural fields the tube wells are spread away from one another and thus operating them becomes very hectic as the person has to run from one place to another to operate the loads. Similar thing happens in our households also. Keeping this thing in mind a system has been designed so that it can ease the farmer as well as save time using the DTMF technology to control the loads remotely.
\end{abstract}

Keywords: DTMF, microcontroller, decoder, Irrigation system, pumps.

\section{Introduction}

The basic idea behind this project is to control the functioning of the agricultural load using wireless technology. In this project we will have two cell phones; one will be handed to the farmer which can send the digital signal to other mobile phone which is normally held in automatic answering mode at the load ends.

At the receiving ends cell phone codes are inputs to the microcontroller, which pre-programmed to identify the command signal coming from the users ends, which is interfaced through relays \& relay drivers according to the desired commands from users end.

Received(April 7, 2017), Review Result(1st: April 28, 2017, 2nd: June 5, 2017), Accepted(July 1, 2017)

${ }^{1}$ Dept. Assistant Professor. Department of Electrical Engineering, THDC Institute of Hydropower Engineering \& Technology, Tehri, Uttarakhand, India

email: mandyguleria20318@gmail.com

${ }^{2}$ Department of Electrical Engineering, THDC Institute of Hydropower Engineering \& Technology, Tehri, Uttarakhand, India email: akkurathi876@gmail.com

${ }^{3}$ Department of Electrical Engineering, THDC Institute of Hydropower Engineering \& Technology, Tehri, Uttarakhand, India email: deepu.sajwan07@gmail.com

${ }^{4}$ Department of Electrical Engineering, THDC Institute of Hydropower Engineering \& Technology, Tehri, Uttarakhand, India email: jsee.negi@gmail.com

${ }^{5}$ Department of Electrical Engineering, THDC Institute of Hydropower Engineering \& Technology, Tehri, Uttarakhand, India email: nidhichau302730@gmail.com 
The cell phones at load site are usually DTMF decoded. DTMF will decode the keywords coming from user's site into digital codes of corresponding frequency which finally fed as input to the microcontroller [1-3].

This gives farmers an ability to press the keypad of the cellphone and can switch on or off the water pumps installed at different positions of the land as per the desired of the farmers. A DTMF decoder \& controlling circuit receives the input commands and control the on - off mode of the connected electrical motor pump [4-6]. This circuit designed is easily available using the various electrical and electronics circuit components. This act as a sign of relief for the areas which comes under draught region, where there is scarcity of rain water such as in Rajasthan. In such areas, a farmer can make better use of limited water and controlling based on weather conditions, environmental conditions. This also helps in water harvesting as water is utilized and not wasted.

\section{Major Hardware Components}

To turn basic the idea of automatic irrigation water pump into realistic state hardware circuit along with software programming is required [7-10].

The major hardware components used in the project are as follow [11-15]:

\subsection{Transformer}

The voltage required by the controlling circuit is of the order up to $20 \mathrm{~V}$ which is available at the output of step down transformer $(240 / 12 \mathrm{v})$. Apart from lowering or raising the voltage, transformers also provide the isolation to low power circuits from high voltage side.

\subsection{Voltage Regulator 7805 IC}

The LM7805 IC is required here to stabilize the fluctuating output of step down transformer to $5 \mathrm{~V}$ constant. The series LM78XX/LM78XXA of voltage regulator is of 3 terminals and available in many constant output voltage levels (0-24V). Key Features-o/p current (0-1000mA), $\mathrm{o} / \mathrm{p}$ voltages $(5-24 \mathrm{v})$ with short circuit and overload protection. 


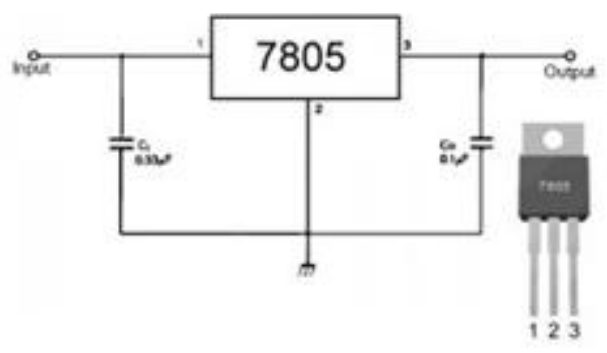

[Fig. 1] LM7805 IC

\subsection{MT8870D DTMF Decoder}

MT8870D is the principle component in the fabrication of this project. DTMF Decoder decodes the dual frequency codes sent by the users from mobile phone and converted signal is fed into the micro controller for required actions. In DTMF technique every digit is represented by two non-integral frequency ranges one is higher and one is lower. As both frequencies are non-integral multiples of each other, it results in correct decoding at the decoder site, even in the presence of non - linearity of filters or harmonics.

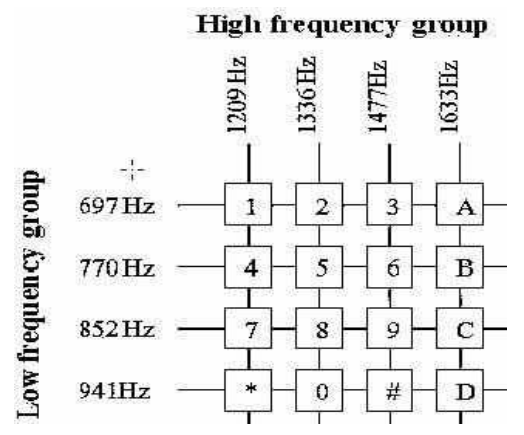

[Fig. 2] DTMF frequency range
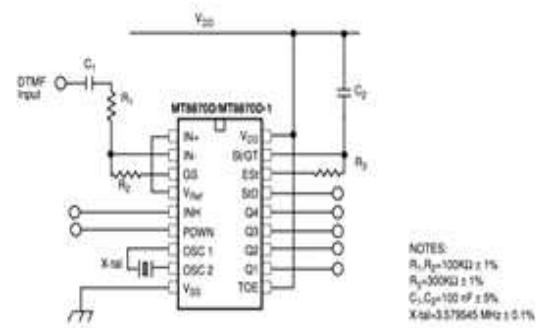

[Fig. 3] DTMF Decoder IC 


\subsection{AT89S52 Microcontroller}

The Microcontroller is the brain of the electronic and controlling circuit. Here in our project as per the requirement we used 8 bit AT89S52 microcontroller. The key feature of the controller is high performance, cost effective to our control applications, energy efficient, easily programmable with $\mathrm{C}$ language, $8 \mathrm{~Kb}$ Flash Memory, RAM 256 bytes, 32 I/O lines, ease of interfacing with computers.

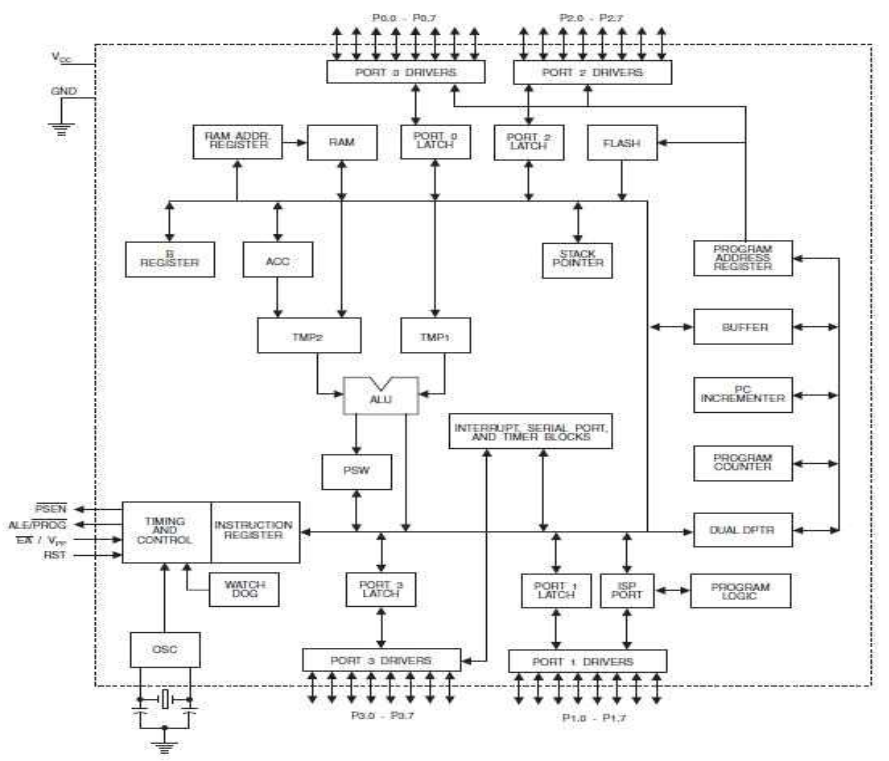

[Fig. 4] AT89S5 Microcontroller

\subsection{ULN 2003A Relay Driver}

The driver used to run relay circuit is ULN2003A. It is connected at the output ports of microcontroller. This driver has the advantage of handling voltage up to $50 \mathrm{v}$ and current to max of 500mA. They are very suitable for turning on high power appliances such as pumps, motor, lightening etc. with isolation to lower power site components.

\section{Working}

As mentioned earlier the receiver's mobile at the load end is connected to the DTMF decoder IC and a call is made from another mobile [7-9].

On forming the network path between two ends, any mobile key is pressed. If 1 is pressed 
then this tone is encoded to 4 digit data signal and fed to micro controller after inverting the data by decoder circuit. The micro controller then will compare the data and output port connected to ULN 2003A driver gets logic high which makes the relay ON. Hence load 1 will be ON.

At the transmitter end, first of all, the farmer will send the DTMF control by first making a call. After the first call is answered then the commands are given by pressing the keys of the user's cell phone, the user will send desired command to switch on/off the motor pumps.

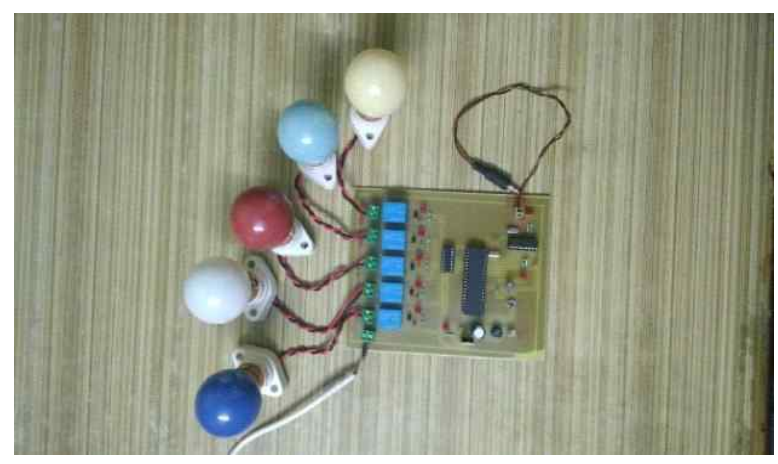

[Fig. 3-1] OFF Mode

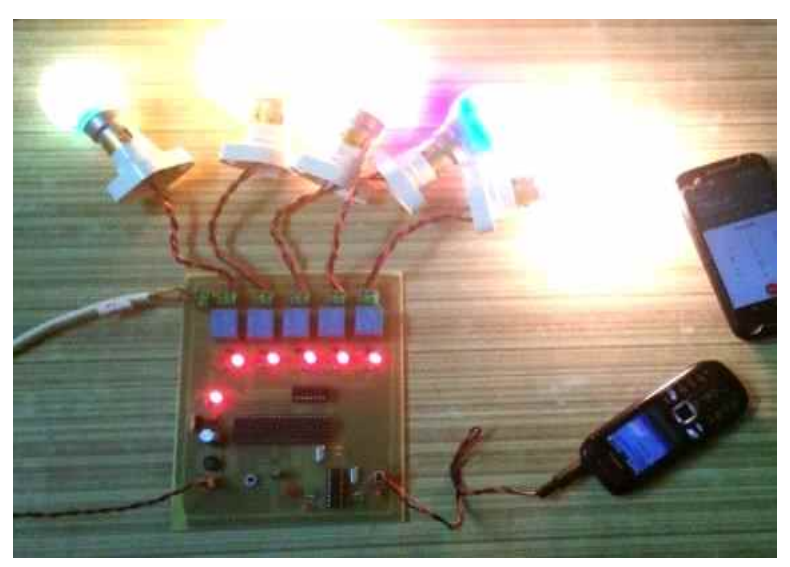

[Fig. 3-2] ON Mode

The command signal received at the load end will be decoded by the MT8870D DTMF decoder control circuit. After the decoding of the signal, a logic controller program is developed in micro controller to recognize \& match the same transmitted cell phone digit corresponding to the DTMF tone, as individual each digit of the cellphone keypad is specify 
by two simultaneous(high or low ) frequency tones selected from a set of frequencies matrix.

A motor driven circuit will then switch on/off the motor pump as per the requirement of the user.

\section{Future Scope}

This project is a transform from the natural irrigation technique which was followed by the farmers to the automatic irrigation which for sure has benefitted in the less wastage of water and also lessens the hard work of the farmers in farms. In future we can plan to add sensors in given project to monitor (i) the humidity level of the soil and (ii) the temperature of the land site which is increased obviously due to fire out in crops, so that healthy measures can be taken immediately to save the farmers from the burden of crops damage. Also this system is used for the home automation, industrial load controlling. The automation system using DTMF and cell phone can be implemented for departmental cabins.

\section{Conclusion}

This project investigates and proposes the controlling of remotely or inaccessible positioned agricultural motor water pumps based on the DTMF technique. This proposed system is designed using discrete components, gates, op-amps. The functionality and working of the entire project circuit is tested and run using simulation software (like MULTISIM) and the complete operational motor switching mode is successfully achieved using the given circuit under consideration. The present project works successfully demonstrated the controlling of remotely located irrigation water pumps for agricultural site without actually going and visiting the site again and again. With this project, system results in achieving adequate water managements due to which there is almost no wastage of water, saves men power, saves time, more efficient.

Further advancements can be done on the practical execution of the proposed irrigation system and to further improve the performance of the system, the feedback closed loop control system will be introduced, where feedback signal is acquired automatically from the agricultural sites using sensors. 


\section{References}

[1] P. P. Mahesh, S. Khismatrao, V. Gadge, A. Thampi, and K. Kalambe, "DTMF Based Agriculture Pump Control," vol. 6, no. 3, pp. 165 - 169, 2016.

[2] Vandana D, Nilesh D And Shailesh C, “ Wireless Sensor Network Based Remote Irrigation Control System And Automation Using Dtmf Code". International Conference on Communication Systems and Network Technologies, pp 34-37,(2011)

[3] International journal of applied engineering research, ISSN 0973- 4562, volume 10, number 12 (2015) pp. 32609-32626 C research India publications.

[4] Tuljappa L, Sanjay L, Sudharshan K, Alok D And Nayan D. "Control Of Remote Domestic System Using Dtmf", Proceedings of ICICI-BME, 69-70. (2009).

[5] Lsmail C and Hamid A, "A Remote Controller For Home And Office Appliances By Telephone". IEEE Transactions on Consumer Electronics, 44(4), 1291-1297. 1998)

[6] Sharma R, Kumar K, and Viq Ss, "Dtmf Based Remote Control System", IEEE International Conference ICIT- 2006; 2380-2383.

[7] V.I. Adamchuk, J.W. Hummel, M.T. Morgan and S.K. Upadhyaya, "On The-Go Soil Sensors for Precision Agriculture", Computers and Electronics In Agriculture, Conference on Science and Engineering, pp. 94-96 (2011).

[8] Rohit S, Kushagra K and Shashank V, "Dtmf Based Remote Control System", IEEE International Conference On Industrial Technology (ICIT), 2380-2383.

[9] Awab Fakih and Jovita Serrao, "Cell Phone Operated Robotic Car", International Journal of Scientific \& Engineering Research, ISSN 2229-5518.

[10] Schenker L, "Pushbutton Calling With A Two- Group Voice-Frequency Code", The Bell System Technical Journal, vol. 39, pp. $235-255,1960$

[11] Justin Cand Rafael Fierro, "Mobile robotic sensors for perimeter detection and tracking", ISA Transactions, 2007; 46(1): 3-13.

[12] http://www.atmel.com

[13] http://www.wikipedia.org

[14] http://www.howstuffworks.com

[15] http://www.alldatasheets.com 